\title{
Orangutan Night-Time Long Call Behavior: Sleep Quality Costs Associated with Vocalizations in Captive Pongo
}

\author{
David R. Samson, ${ }^{1}$ Del Hurst, ${ }^{2}$ and Robert W. Shumaker ${ }^{2,3,4}$ \\ ${ }^{1}$ The Nunn Lab, Department of Evolutionary Anthropology, Duke University, Durham, NC 27708, USA \\ ${ }^{2}$ Indiana University, E. Kirkwood Avenue 701 Student Building 130, Bloomington, IN 47405-7100, USA \\ ${ }^{3}$ Indianapolis Zoo, West Washington Street 1200, Indianapolis, IN 46222-0309, USA \\ ${ }^{4}$ Krasnow Institute at George Mason University, Fairfax, VA 22030, USA
}

Correspondence should be addressed to David R. Samson; drsamson@gmail.com

Received 13 June 2014; Revised 28 July 2014; Accepted 11 August 2014; Published 7 September 2014

Academic Editor: Luciano J. Avila

Copyright (C) 2014 David R. Samson et al. This is an open access article distributed under the Creative Commons Attribution License, which permits unrestricted use, distribution, and reproduction in any medium, provided the original work is properly cited.

\begin{abstract}
Researchers have suggested that the ability of male primates to emit long-distance vocalizations is energetically costly and potentially incurring important adaptive consequences upon the calling individuals. Here, we present the first preliminary data on captive orangutan (Pongo spp.) nocturnal long calls, generated at the Indianapolis Zoo. We used videography to characterize long calls with observed behavioral contexts for 48 nights ( 816 observed hours totaling 83 long calls). We generated somnographic data for a subset of the long calls. Overall measures of sleep quality generated by infrared videography were then compared to the somnographic, nocturnal long call data. We tested hypotheses related to the proximate mechanisms involved in the initialization of vocalization and the potential costs of emitting long calls to overall sleep quality. We found that (1) performed long calls were conscious and premeditated in nature and (2) greater number of night-time long calls shared a positive relationship with arousability and sleep fragmentation and a negative relationship with total sleep time and sleep quality. These findings strongly suggest that only several minutes of total time invested in long calls throughout the night disproportionately cost the caller by negatively impacting overall sleep quality.
\end{abstract}

\section{Introduction}

Several nonhuman primate species are known to emit "loud calls." These alarm call vocalizations are like most animal acoustic signals in that they are primarily produced during their active period [1-6] and thus are characterized by species-specific circadian distribution $[7,8]$. Given primate loud calls are stereotypically characterized by traits such as acoustic intensity $(\mathrm{dB})$, form type (i.e., length frequency modulation), and often vocalized by high ranking males [9], it has been suggested that Old World monkey and ape loud calls are phylogenetic homologous characteristics [10]. In general, several hypotheses have been forwarded in attempts to explain the function of primate loud calls: mate-attraction, inter-group spacing and intra-group cohesion, and territorial advertisement [11].
Within the repertoire of orangutan (Pongo) vocalizations the long call is characterized by several idiosyncratic traits: it is produced often by large individuals [5, 12-14], it travels greater than 300 meters [15], it has only been observed to be emitted by flanged males $[13,14,16]$, it is the loudest call in the repertoire (reaching $100 \mathrm{db}$ at 1 meter away and can be heard up to more than $1 \mathrm{~km}$ in distance) and calls may exceed three minutes in length $[13,17]$.

It has been hypothesized that in the wild long calls attract adult females to coordinate mating [5, 12, 14, 18], act as a mechanism with which individuals associate within a community and coordinate seasonal movements $[19,20]$ and/or mediate dominance relationships among adult males $[5,21,22]$. Specific functions have been proposed, including signaling nulliparous females to initiate mating with dominant males $[12,23,24]$ and/or serve as a beacon for 
location for sexually receptive females given long distances $[12,25,26]$, keeping potentially antagonistic males apart [5, $12,14,27]$, and a way for males to communicate their next day travel direction [28]. It has also been suggested these calls may function as a social mechanism to restrict hormone production in small subordinate/unflanged males and thus keep them from developing into large dominate flanged males, although this hypothesis has yet to be tested [29].

The proximate mechanisms and/or stimuli involved in the initiation of long calls remain unknown. MacKinnon (1974:54) noted: "Sometimes the calls seemed spontaneous but often they followed a sudden sound cue. Calls were. . .triggered by sudden pig noises. . .the bark of a deer. . .a human sneeze. . a distant gunshot... a sudden gust of wind and a clap of thunder...the crash of nearby tree-fall...or the sound of breaking branches." Furthermore, RS has noted anecdotal evidence that Azy (the flanged male observed in this captive group) has emitted long calls in response to spontaneous environmental cues (e.g., automobiles driving by, wind gusts, etc.). The REM (rapid eye movement) sleep state is associated with vivid dreaming; during sleep dreaming occurs because the brain attends to endogenously generated activity which can be internally perceived as actually occurring events-much like hallucination [30]. Therefore it is possible that sleep-to-wake transitions leading to long calls could be catalyzed from an endogenous stimulus. Quantitative data circumscribing the context with which captive orangutans perform long calls has yet to be recorded.

In mammals (humans included), sleep fragmentation has been found to diminish attention, sensory-motor processing, motivation, and memory [31-34]. These diminishing effects in behavioral performance have been associated with direct costs to the individual [35]. The costs of sleep deprivation on waking function are well documented, yet contextual descriptions in which sleep loss is adaptive are emerging. Costly morphological, physiological, and behavioral traits have been evolutionarily selected, especially via sexual selection [36]. In an extreme example, the polygynous pectoral sandpiper has been found to remain active for greater than $95 \%$ of a 19 day period when females are in peak fertility [37]. To date, it has yet to be assessed whether orangutans experience any physiological costs to sleep quality relative to the investment directed in night-time long call performance.

The goal of this study was to document and describe captive orangutan night-time vocalizations. In addition, hypotheses related to (1) the proximate mechanisms involved in the initialization of vocalization and (2) the potential costs of emitting long calls to overall sleep quality were tested. First, we hypothesize that night-time long calls will begin from an abrupt sleep-to-wake transition, as an unconscious reactionary response to abiotic forces (e.g., loud, disruptive noises) or internal sleep states (i.e., dreams). Second, we hypothesize that individuals that vocalize a greater number of long calls will experience reduced overall sleep quality. Specifically, we tested the following predictions.

(1) Night-time long calls will initialize from a REM stage of sleep and/or in association with high-decibel environmental stimulus.
(2) Greater number of night-time long call vocalizations will reduce sleep quality and sleep duration and increase sleep arousability (number of motor activity bouts per hour) and fragmentation (the number of brief awakenings greater than 2 min per hour).

\section{Methods}

2.1. Study Subjects. Subjects housed at the Indianapolis Zoo (total $N=5$ ) were three females, Katy (Studbook ID number: 2248), Knobi (1733), and Lucy (1972), and two males Azy (1616) and Rocky (3331). All subjects were classified as adults with the exception of Rocky, the only adolescent. None of the subjects were geriatric, as life span in the wild for orangutans is approximately 60 years old [38]. All subjects were hybrids of Bornean (Pongo pygmaeus) and Sumatran (Pongo abelii) species. Rocky, Katy, and Lucy were privately owned and were part of the entertainment industry prior to moving into the Association of Zoos \& Aquariums (AZA) community; specific information about their personal histories is therefore limited (RS personal data). The individuals from the entertainment industry were hand-reared by humans, none having any exposure to their mothers during early growth and development. Azy and Knobi have always lived within the AZA community and have well documented biographies and rich social experience. Subjects were housed in interconnected indoor and outdoor enclosures and had regular access to all areas throughout the duration of the study. The indoor enclosure contained laminate sleeping platforms located approximately $1 \mathrm{~m}$ off the floor. The indoor space included five possible sleeping rooms. Subjects had access to natural and artificially enriched environments. The indoor enclosure was set at a constant temperature of $23.3^{\circ} \mathrm{C}$. Natural lighting was the primary source of light for the group and was accessible by way of windows and access to the outdoor enclosure; in addition, lights were manually turned on by the keepers at $07: 30 \mathrm{~h}$ and turned off at 17:30 h. For further detail regarding night-time sleep related behaviors in captive orangutans see [39-41].

2.2. Data Collection. This study was conducted over four months during August 2012-November 2012. The occurrences of long calls were continuously video-recorded nightly, from 16:00-09:00 for a total of 48 nights ( 816 hours total). Alloccurrence sampling captured each instance of vocalization throughout the nightly period (total $N=83$ ). The temporal distributions of long calls were tabulated to describe occurrence of calls associated within hourly intervals. Context of long calls was recorded; nominal data were generated for vocalization instances, such as the presence or absence of associated copulation, presence or absence of discrete abiotic (i.e., automobiles and inclement weather, etc.) or biotic (i.e., vocalizations by conspecifics) noises, direction (i.e., vocalizing into the wall or directed towards conspecifics), stationary versus mobile (i.e., states were defined as mobile if the vocalizer moved out of the sleep area during the long call), and state prior to vocalization (i.e., upright awake, resting awake, or sleep). 


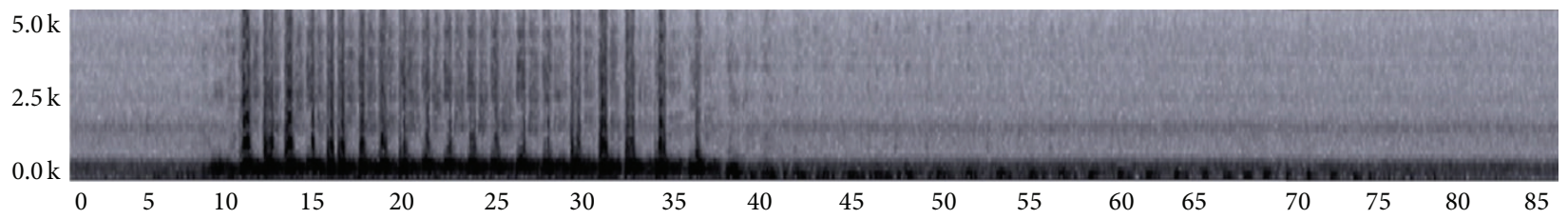

FIgURE 1: An example audio-spectrogram of a night-time long call produced by Azy. Notice the tail-off is long and the frequency is lower than the human range of ability to hear.

Sleep behavior was recorded continuously throughout the night using all-occurrence sampling on subjects [42]. Two instruments (AXIS P3344 and AXIS Q6032-E Network Cameras) were used to generate nightly sleep quota data on subjects within line of sight. One stationary camera (P3344) was manually placed in front of the subject at the time of sleeping platform construction; another rotatable camera (Q6032-E) was remotely controlled throughout the night to ensure focal subjects were continuously within line of sight from start to finish of the recording session (Axis Communications, Lund, Sweden). Videography generated values for sleep quotas (for detailed methods on sleep behavior analysis see [40]): total time spent awake, total NREM (nonrapid eye movement), total REM, total sleep time (sum of NREM and REM), and total time in bed (absolute difference between rising and retiring times from their constructed sleeping platforms). Measures of overall sleep quality include sleep fragmentation (the number of brief awakenings greater than 2 min per hour), arousability (number of motor activity bouts per hour), and sleep quality (sleep duration/time in bed).

Long calls were recorded using an infrared camera (P3344 Axis with a two-way built in mic) and then converted from asf into .wav files for audio analysis. Detailed audiospectrographic analysis was performed on 29 long calls. All sound analyses were conducted using Audacity acoustic analysis computer program (Audacity 1.3.12-beta). Audio data generated included minimum frequency (the number of times that a periodic vibration occurs within a 1 second period measured in $\mathrm{Hz}$ ), maximum frequency, duration (total time of vocalization), peak frequency (the greatest instantaneous value of a standard frequency), and peak decibel (a ratio between the measured level and a reference threshold level indicative of acoustic power, measured in $\mathrm{dB}$ ). All minimum frequency measures were taken at the end of the long call vocalizations, as this is where the lowest frequencies were thought to occur. Peak frequency, maximum frequency, and peak $\mathrm{dB}$, however, were obtained from analysis of the entire call.

2.3. Data Analysis. We generated descriptive statistics characterizing the nightly distribution of call frequency and duration (statistical tests were conducted using IBM SPSS 21); average calls per night and average number of calls per observation hour were calculated. We generated long call values from audio-spectrographic analysis, which were checked for normality with Kolmogorov-Smirnov tests. Frequencies for nominal categories were generated and $\chi^{2}$ was adopted to test expected versus observed frequencies of sleep/wake

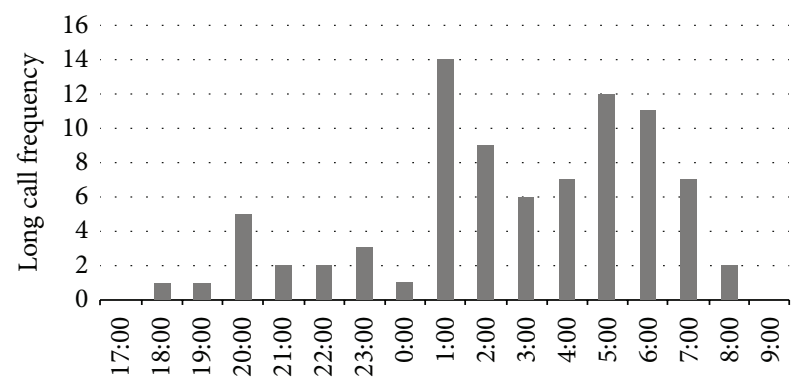

FIGURE 2: The occurrence of adult male long calls was recorded at all hours from 17:00-09:00. The circadian distribution of calls revealed a bimodal pattern. Bimodal long call frequency is characteristic of orangutan male vocalizations in the wild but is in this instance of captivity expressed in a temporally unique manner.

states prior to long calls (2-tailed) and temporal distribution of long calls. Independent-samples $t$-test was used to compare the intensity of long calls between states and the difference between high occurrence vocalization nights (i.e., nights where long calls were greater than 2) versus low occurrence vocalization nights (i.e., nights where long calls were less than 3) determined by using the midway point of the call range distribution. We applied Spearman's rho correlation (due to nonnormality in analyzed variables) coefficients $(r)$ to examine relationships among long calls emitted and overall sleep quality (1-tailed); we include correlation slopes. All reported errors are standard deviations and all tests set at the significance level of $P \leq 0.05$.

\section{Results}

Results for this study show that only one of the five subjects vocalized long calls (the fully flanged male named Azy). The long calls (see Figure 1, e.g., spectrograph) were similar in structure to previously described wild long calls [5] and generally consisted of a traditional three part structure (introduction, climax, and tail-off); although as noted by other researchers [17] deviations from the three-part structure are known to occur.

Azy long-called $1.73 \pm 1.00$ times per night and averaged $0.10 \pm 0.09$ long calls per survey hour. The long call temporal distribution (Figure 2) shows significant differences in expected (with the assumption that occurrence of long calls would be equal per hour of observation) and observed distributions $\left(\chi^{2}=62.0, d f=16, P<0.01\right)$; there was a bimodal distribution, with two peaks approximately at 01:00 (total 


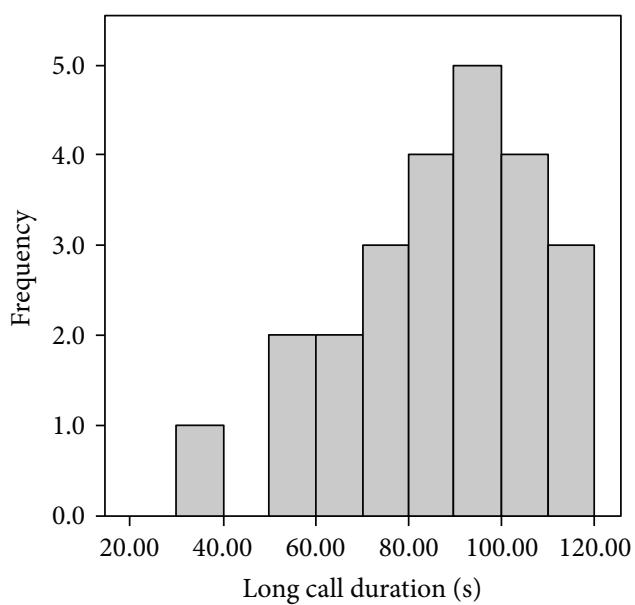

(a)

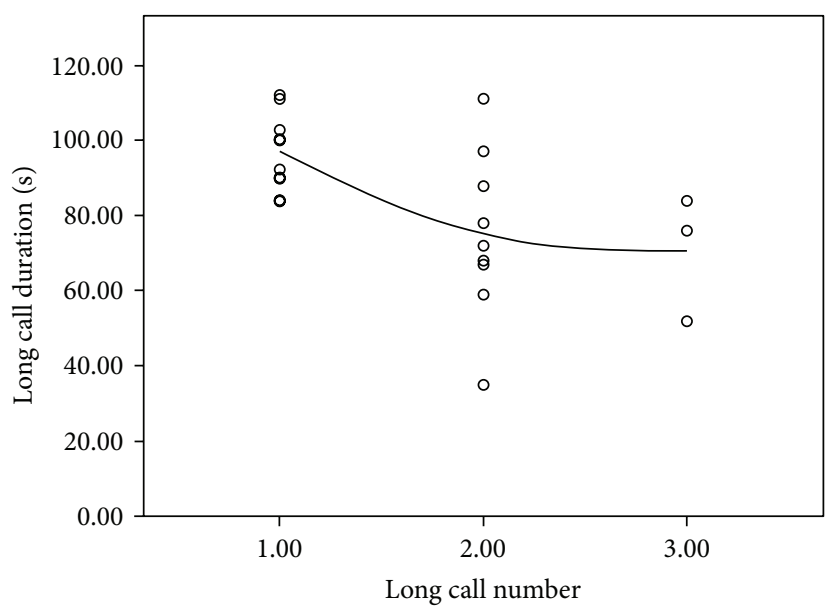

(b)

Figure 3: (a) A histogram of male orangutan long call duration; duration was normally distributed. (b) Long call duration decreased with each additional call throughout the night.

TABLE 1: Descriptive statistics characterizing night-time flanged male orangutan long calls $(n=29)$ generated from somnographic analysis.

\begin{tabular}{lccc}
\hline & Range & Mean \pm SD & Test of normality \\
\hline Long call duration & $35.0-112.0$ & $85.4 \pm 19.5$ & $P=0.20$ \\
Long call min. frequency & $12.0-59.0$ & $19.5 \pm 10.1$ & $P<0.001$ \\
Long call peak frequency & $59.0-400.0$ & $278.4 \pm 93.5$ & $P=0.009$ \\
Long call peak dB & $-35.0-0.0$ & $-10.0 \pm 12.4$ & $P=0.012$ \\
\hline
\end{tabular}

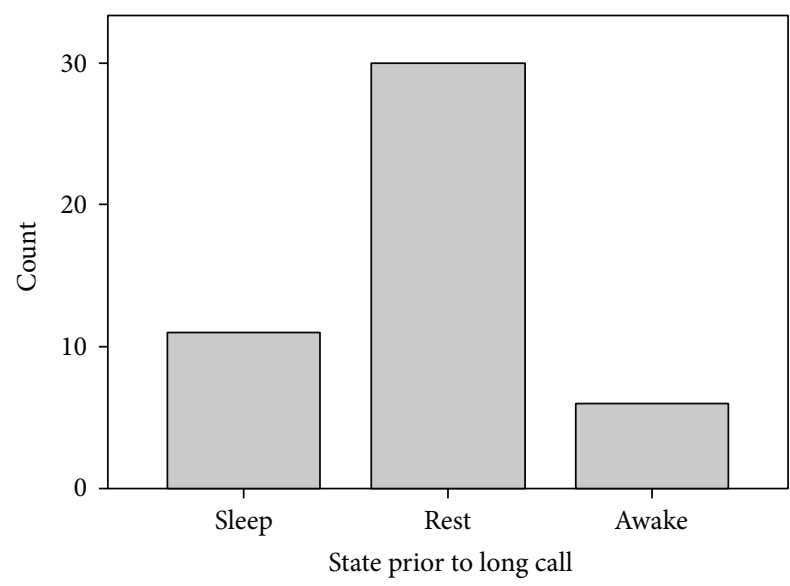

Figure 4: Azy most often called prior to several minutes of restful alertness, suggesting calls were premeditated and conscious.

vocalizations, $N=14$ ) and 05:00 (total vocalizations, $N=$ 12 ) in the morning. Spectrographic analysis of the long calls $(N=29)$ shows the average long call duration to be $85.4 \pm 19.5$ seconds (see Table 1 and Figures 3(a) and 3(b)). Furthermore, long calls significantly decreased in duration with each additional long call $\left(r^{2}=-0.64, P=0.001\right.$; Figure 3(b)).

Prelong call context and state was shown to be predominantly from an awake but restful state (Figure 4). Observed prelong call state tested against expected occurrence (a previous study resulted in $72 \%$ of the time in a sleeping area to be spent asleep [42]) shows that Azy was in a significantly different state than expected (sleep state $23 \% ; \chi^{2}=119.01$, $d f=1, P<0.001)$. Of the 11 times Azy was in a sleep state prior to long calls, he was in REM state only twice $(4.2 \%$ of the overall sample). Azy long-called directed at conspecifics $67.4 \%$ of the time, whereas he called directly into the wall or a walled corner $30.4 \%$ of the time $(2.2 \%$ he was out of line of site and direction could not be determined). Long calls directed at conspecifics did not differ in peak $\mathrm{dB}(N=19,-19.7 \pm 11.2$ versus $N=12,-13.3 \pm 12.0$; independent-samples $t$-test, $t=$ $-1.5, P=0.14)$ or peak frequency $(N=15,277.9 \pm 90.0$ versus $N=7,322.1 \pm 58.1$; independent-samples $t$-test, $t=-1.19$, $P=0.25)$, when compared to long calls directed into walls. Azy was stationary $67.4 \%$ and was mobile $30.4 \%$ of the time during long calls. The moments before initiation of long calls were analyzed for discrete abiotic or biotic noises; no such instances were observed. A copulation was associated with a long call only once (1.7\%); no associated copulation was observed a majority of the time $(55.2 \%)$; although he was outside the line of sight for 13 instances, therefore, associated copulation cannot be ruled out for these instances.

Several measures of sleep quality significantly reduced relative to the number of nightly long calls (Table 2 and Figures 5(a) and 5(b)). As the number of nightly long calls performed by Azy increased, his arousability increased, sleep fragmentation increased, sleep quality decreased, and total time spent asleep decreased (see Figure 6 illustrating performed long call posture and intent). When compared to nights that had a low number of total calls (less than 3 


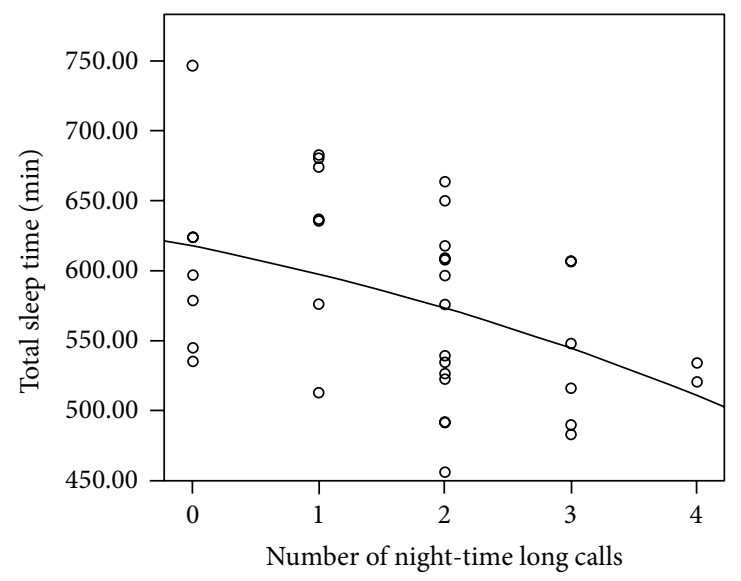

(a)

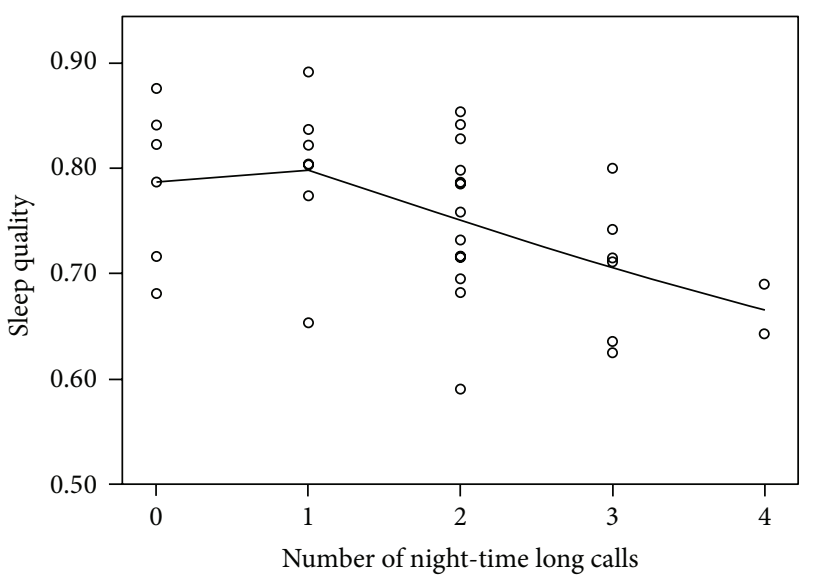

(b)

Figure 5: (a) Azy experienced less nightly total sleep time and (b) sleep quality the more he invested in performing long calls. The correlation slope for total sleep time and number of night-time long calls was $Y=0.81 \pm 0.03$; the interpolation line used a quadratic fit method. The correlation slope for sleep quality and number of night-time long calls was $Y=6.22 E 2 \pm 25.68$; the interpolation line was linear.

TABLE 2: Spearman's rho correlation showing the significant relationship between the number of nightly long calls $(n=35)$ and measures of sleep quality: arousability (number of motor activity bouts per hour), sleep fragmentation (the number of brief awakenings greater than 2 min per hour), sleep quality (sleep duration/time in bed), and total sleep time (mins).

\begin{tabular}{|c|c|c|c|c|c|c|c|c|}
\hline & \multicolumn{2}{|c|}{ Arousability } & \multicolumn{2}{|c|}{ Sleep fragmentation } & \multicolumn{2}{|c|}{ Sleep quality } & \multicolumn{2}{|c|}{ Total sleep time } \\
\hline Number of nightly long calls & $r^{2}=0.31$ & $P=0.35$ & $r^{2}=0.30$ & $P=0.04$ & $r^{2}=-0.47$ & $P=0.002$ & $r^{2}=-0.44$ & $P=0.004$ \\
\hline
\end{tabular}

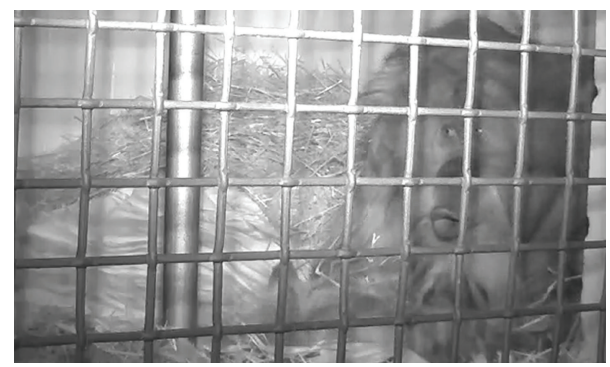

FIGURE 6: Azy performing a stationary long call from a state of restful alertness; the vocalization was directed at conspecifics and not associated with a copulation.

vocalizations) high total long call nights (greater than 2 vocalizations) were associated with significantly decreased sleep quality $(N=27,0.77 \pm 0.07$ versus $N=8,0.70 \pm 0.06$; independent-samples $t$-test, $t=2.26, P=0.012$ ).

\section{Discussion}

To our knowledge, this study is the first to describe nighttime long calls in captive orangutans; captive and wild environments differ in several ways which could affect long call behavior-notably, captive settings control for proximity to conspecifics and a consistent rest/wake period. Azy, the lone fully flanged male, was the only individual to perform long call vocalizations which is consistent with observations in the wild [5]. Azy's long call pattern exhibits structure previously described by researchers (see supplemental video in Supplementary Material for long call example available online at http://dx.doi.org/10.1155/2014/101763) [5, 19, 43]. Azy produced exhalation as well as inhalation sounds, commonly expressed by an exhalation bubbling and then hiatus roars and intermediaries and then a long trailing of sighs [17]. Long call nightly temporal distribution fit a bimodal pattern, which is characteristic of male orangutans circadian distribution in the wild [7]; interestingly, although the distribution was bimodal, it temporally did not correspond to any known pattern exhibited in the wild. Azy most frequently performed nocturnal long calls between the hours of 01:00-02:00 and 05:00-06:00. Azy's peak calling time fit with the predawn long call rates seen in wild populations, but his greatest number of long calls was between 01:00-02:00-in stark contrast to a study performed at Batang Ai National Park in Northern Borneo, which found no recorded instance of a long call during this period [7]. Finally, the average calls per survey hour heard at Batang Ai were 0.45, whereas Azy's call per survey hour were 0.10 ; this is most likely due to a lack of long call response behavior to potentially antagonistic males, given no other adult males were present.

The duration of long calls reduced throughout the night (Figure 3(b)). We hypothesize this could either be related to increasing fatigue associated with multiple long call performances or an association with differing levels of alertness related to the passage of SWS dominated early sleep and increasingly lengthening REM stages towards the end of the night [44], assuming a human-like pattern in orangutans. 
Quantitative assessment of the energy costs and proposed fatigue buildup of sequential long calls waits experimental testing.

The first hypothesis, that night-time long calls will begin from an abrupt sleep-to-wake transition, as an unconscious reactionary response to abiotic forces or internal sleep states, was rejected. Azy's prelong call state can be characterized as a several minute period of alert-restfulness. Two instances were observed when long calls were initiated from a sleep state ( $\sim 4 \%$ of the overall sample) and were associated with REM sleep stages; his observed behavior was not characterized by abrupt, reactionary movements. Furthermore, Azy directed long calls $\sim 67 \%$ of the time towards conspecifics, which is suggestive of the premeditated intention in this behavior, although intentionality in animal communication is difficult to assess; an alternative interpretation is that postural orientation was by chance, rather than intentional. We hypothesized that wall directed calls function as acoustic amplification; testing vocalization peak $\mathrm{dB}$ and frequency between both direction states (towards conspecifics versus towards a wall) revealed no difference between the outputs. Although, it could be that wall directed long calls appear to be more acoustically powerful to the individual caller, despite the fact that there was no overall difference in $\mathrm{dB}$ levels to others within the enclosure. Azy long-called from a stationary, upright position a majority of the time $(\sim 67 \%)$. Only a single copulation was observed as associated with a long call; this is not suggestive of the function of long calls in a captive context initiating sex; it should be noted that $\sim 28 \%$ of post long call sample was out of line of sight due to Azy leaving his sleeping area during mobile long call displays. Additionally, female estrous could have exerted a possible influence on copulation behavior, but these states were not recorded. Therefore we are cautious against interpretation rejecting or supporting this conclusion. Overall, we interpret this data as supporting evidence for the supposition that long calls performed by Azy may be conscious and premeditated in nature.

The second hypothesis, that individuals that vocalize a greater number of long calls will experience reduced overall sleep quality, was supported; greater number of night-time long calls elicited by Azy were associated with poorer sleep quality. Greater occurrence of long calls share a positive relationship with arousability and sleep fragmentation and a negative relationship with total sleep time and sleep quality. The average long call was 85 seconds: therefore, the total time needed to be in a wake state is marginal relative to the total time spent asleep (i.e., a night with two total long calls costs 2.8 minutes of sleep ( $0.005 \%$ total time asleep), whereas a night with four total long calls costs 5.6 minutes $(0.01 \%$ total time asleep)). Despite this marginal total duration of time invested in long calls, the effect on sleep quality is significant (a decrease in $9 \%$ of the total proportion of time asleep relative to the time spent in the sleeping area). We interpret this data as being evidence of a significant investment of energy on the part of the caller; this could manifest in the cognitive preamble before a long call or the cool down cost associated with postlong call excitement. Yet, we acknowledge that causal direction remains unclear; for example, it could be that, during restive nights, the individual is conscious longer and therefore is more prone to exhibit long call behavior. One way forward may be the monitoring of night-time long call investment relative to the hormonal profiles of not only individuals that call, but individuals that are within audio range of the caller. Given the proximate mechanisms for this phenomenon are poorly understood, we suggest future research should investigate the endocrinological correlates of this behavior.

The comparison of a trait in both wild and captive contexts is beneficial in that the trait in question can be observed in a controlled environment, removed from intervening variation. Some insights can be made, from observing the context of captive long call behavior in orangutans. We tested the proximate stimulus of external or endogenous "surprise" cues to explain spontaneous long calls-which our data rejected. There is no evidence to suggest that long calls in a zoo environment serve functionally as postsleep travel planning. An evolutionary (intrasexual competition) cause is less relevant because there are no other adult males in the enclosure. Reproductive fitness could be directly associated with long calls, but the evidence from this study is equivocal given that only $1.7 \%$ of observed calls were associated with mating. Furthermore, this behavior in Azy could simply be a manifestation of ontogenetic factors if he was exposed to his father's vocalizations during development, but unfortunately there is no evidence that his father performed night-time long calls. Yet, there is significant cost in sleep quality associated with greater investment in night-time long calls, which may have adverse effects on next day cognition [39]; the persistence of such costly behavior, in a controlled context where the benefits of the behavior are less obvious, indicates that it is likely to be functionally adaptive, although we are cautious that interpreting behavior based on current ecology relative to an evolved function presents obvious difficultiesas it would take several generations to lose a behavior not under selective pressure. Finally, it should be noted that the sample is one individual, which may not be statistically representative of Pongo; notwithstanding; this data reveals the capability of the species [45] and awaits further confirmation with captive flanged, male Pongo at other institutions.

The cross-cultural study of human sleep expression, sleep architecture (distribution of NREM and REM throughout a nightly sleep bout), diurnal bouts of inactivity (i.e., napping and/or energy conservation), and sleep quality is in its infancy [46-49]. The sociophysical ecology of human sleep is more readily accessible in historical and ethnographic records, yet the only data relative to forager sleep expression is anecdotal. It has been noted that the forager pattern may be polyphasic $[50,51]$; even preindustrial, preelectric western populations divided the night into "first sleep" and "second sleep," indicating a polyphasic pattern [52]. Chimpanzees night-time vocalizations at Mahale have been recorded to be especially active during the periods of 23:00-02:00, with a predominance of pant-hoots associated with night-time defecation and urination [53]. With respect to orangutans, it may also be the case that the cost of night-time vocalizations can be made up during the day with "siesta napping," 
which has been observed in all ape populations [54] and is characteristic of equatorial forager's daily inactivity patterns $[55,56]$.

In conclusion, captive male orangutans exhibit long call behavior which can be characterized with relatively the same form and structure as their wild counterparts. Given the evidence for an alert preamble to long calls, these findings suggest that the behavior may have been conscious and premeditated in nature. Furthermore, only several minutes invested in long calls throughout the night disproportionately cost the caller by negatively impacting overall sleep quality. The fact that this behavior persists in a captive environment, where the benefits for the behavior are less obvious, may indicate that the ability is adaptive in many wild social and ecological conditions. In polygynous species, in which paternal investment in offspring is minimal to absent, access to fertile females is essential to male reproductive fitness; although researchers have yet to unravel the function of nocturnal long calls in wild populations, it may be that sexual selection favors an ability in males to forgo sleep or experience lower levels of sleep quality, when overall reproductive benefits outweigh the cost of the behavior. We therefore do not expect such sleep quality-to-costs tradeoffs to be limited to orangutans but rather to exist also in other primates as well.

\section{Conflict of Interests}

The authors declare that there is no conflict of interests regarding the publication of this paper.

\section{Authors' Contribution}

David R. Samson and Robert W. Shumaker designed research; David R. Samson and Del Hurst performed research; David R. Samson and Del Hurst analyzed data; and David R. Samson wrote the paper.

\section{Acknowledgments}

The authors thank the staff at the Indianapolis Zoo, especially Paul Grayson, David Hagan, Lisa Goodwin, John Pilarski, and Chad Harmon. The authors would like to thank Stephanie Dickinson and the Indiana Statistical Consulting Center (ISCC) for providing statistical support. The authors gratefully acknowledge funding this research provided by the following institutions: The Indiana Academy of Science, The American Society of Primatologists, and Sigma Xi. Data collection protocol was reviewed and approved by the Indiana University Institutional Animal Care and Use Committees (IACUC).

\section{References}

[1] D. J. Chivers, "An introduction to the socio-ecology of Malayan forest primates," in Comparative Ecology and Behaviour of Primates, R. Michael and J. H. Crook, Eds., pp. 101-146, Academic Press, London, UK, 1973.

[2] T. Dabelsteen and N. Mathevon, "Why do songbirds sing intensively at dawn? A test of the acoustic transmission hypothesis," Acta Ethologica, vol. 4, no. 2, pp. 65-72, 2002.
[3] T. Geissmann and T. Mutschler, "Diurnal distribution of loud calls in sympatric wild indris (indri indri) and ruffed lemurs (Varecia variegata): implications for call functions," Primates, vol. 47, no. 4, pp. 393-396, 2006.

[4] A. Keast, "Temporal vocalisation patterns in members of a eucalypt forest bird community: the effects of weather on song production," Emu, vol. 94, no. 3, pp. 172-180, 1994.

[5] J. Mackinnon, "The behaviour and ecology of wild orangutans (Pongo pygmaeus)," Animal Behaviour, vol. 22, no. 1, pp. 3-74, 1974.

[6] C. A. Staicer, D. A. Spector, and A. G. Horn, "The dawn chorus and other diel patterns in acoustic signalling," in Ecology and Evolution of Acoustic Communication in Birds, D. E. Kroodsma and E. H. Miller, Eds., pp. 426-453, Cornell University Press, Ithaca, NY, USA, 1996.

[7] M. D. Ross and T. Geissmann, "Circadian long call distribution in wild orangutans," Revue de Primatologie, vol. 1, pp. 1-10, 2009.

[8] R. M. Seyfarth, "Vocal communication and it relation to language," in Primate Societies, B. B. Smuts, D. L. Cheney, R. W. Wrangham, and T. T. Struhsaker, Eds., pp. 440-451, The University of Chicago Press, Chicago, Ill, USA, 1987.

[9] P. M. Waser, "The evolution of male loud calls among mangabeys and baboons," in The Neglected Ape, S. T. Snowdon, C. H. Brown, and M. R. Petersen, Eds., pp. 67-93, Cambridge University Press, Cambridge, UK, 1982.

[10] T. Geissmann, "Gibbon songs and human music from an evolutionary perspective," in The Origins of Music, N. L. Wallin, Ed., pp. 103-123, The MIT Press, Cambridge, Mass, USA, 2000.

[11] G. Hohmann and B. Fruth, "Loud calls in great apes: sex differences and social correlates," in Current Topics in Primate Vocal Communication, E. Zimmermann, J. D. Newman, and U. Jurgens, Eds., pp. 161-184, Plenum Press, New York, NY, USA, 1995.

[12] B. M. F. Galdikas, "The orangutan long call and snag crashing at Tanjung puting reserve," Primates, vol. 24, no. 3, pp. 371-384, 1983.

[13] J. R. MacKinnon, The Behaviour and Ecology of the Orangutan (Pongo pygmaeus) with Relation to other Apes, Oriel College, Oxford, UK, 1972.

[14] J. C. Mitani, "Sexual selection and adult male orangutan long calls," Animal Behaviour, vol. 33, no. 1, pp. 272-283, 1985.

[15] A. R. Lameira and S. A. Wich, "Orangutan long call degradation and individuality over distance: a playback approach," International Journal of Primatology, vol. 29, no. 3, pp. 615-625, 2008.

[16] R. A. Delgado, A. R. Lameira, M. D. Ross et al., "Geographical variation in orangutan long calls," in Orangutans: Georgraphic Variation in Behavioral Ecology and Conservation, A. A. Wich, S. S. Utami Atmoko, T. Mitra Setia, and C. P. van Schaik, Eds., pp. 215-224, Oxford University Press, New York, NY, USA, 2009.

[17] M. D. Ross and T. Geissmann, "Call diversity of wild male orangutans: a phylogenetic approach," The American Journal of Primatology, vol. 69, no. 3, pp. 305-324, 2007.

[18] J. MacKinnon, "Reproductive behavior in wild orangutan populations," Perspectives on Human Evolution, vol. 5, pp. 257-273, 1979.

[19] R. A. Delgado Jr., "Geographic variation in the long calls of male orangutans (Pongo spp.)," Ethology, vol. 113, no. 5, pp. 487-498, 2007.

[20] B. M. G. Galdikas, "Adult male sociality and reproductive tactics among orangutans at Tanjung Puting," Folia Primatologica, vol. 45, pp. 9-24, 1985. 
[21] R. A. Delgado Jr. and C. P. van Schaik, “The behavioral ecology and conservation of the orangutan (Pongo pygmaeus): a tale of two islands," Evolutionary Anthropology, vol. 9, no. 5, pp. 201218,2000 .

[22] I. Singleton and C. P. van Schaik, "The social organisation of a population of Sumatran orang-utans," Folia Primatologica, vol. 73, no. 1, pp. 1-20, 2002.

[23] C. Schurmann, "Mating behaviour of wild orangutans," in The Orangutan: Its Biology and Conservation, L. de Boer, Ed., pp. 269-284, Dr. W. Junk, The Hague, The Netherlands, 1982.

[24] B. F. M. Galdikas, "Social and reproductive behavior of wild adolescent female orangutans," in The Neglected Ape, R. D. Nadler, L. L. Sheeran, and N. Rosen, Eds., Plenum Press, New York, NY, USA, 1995.

[25] D. A. Horr, “The Borneo orang-utan," Borneo Research Bulletin, vol. 4, pp. 46-50, 1972.

[26] D. A. Horr, "The Borneo orang-utan: population structure and dynamics in relationship to ecology and reproductive strategy," in Primate Behavior, L. A. Rosenblum, Ed., pp. 307-323, Academic Press, New York, NY, USA, 1975.

[27] H. D. Rijksen, A Field Study on Sumatran Orangutans (Pongo Pygmaeus Abelli, Lesson 1827), 1978, edited by H. Veenman and Z. Wageningan.

[28] C. van Schaik, L. Damerius, and K. Isler, "Wild orangutan males plan and communicate their travel direction one day in advance," PLoS ONE, vol. 8, pp. 1-10, 2013.

[29] A. N. Maggioncalda, "Male orangutan development and reproduction," in Orangutan Species Survival Plan Husbandry Manual, pp. 32-35, 1997.

[30] M. W. Mahowald, S. R. Woods, and C. H. Schenck, "Sleeping dreams, waking hallucinations, and the central nervous system," Dreaming, vol. 8, no. 2, pp. 89-102, 1998.

[31] D. Dawson and K. Reid, "Fatigue, alcohol and performance impairment," Nature, vol. 388, article 235, 1997.

[32] J. S. Durmer and D. F. Dinges, "Neurocognitive consequences of sleep deprivation," Seminars in Neurology, vol. 25, no. 1, pp. 117-129, 2005.

[33] A. Rolls, D. Colas, A. Adamantidis et al., "Optogenetic disruption of sleep continuity impairs memory consolidation," Proceedings of the National Academy of Sciences of the United States of America, vol. 108, no. 32, pp. 13305-13310, 2011.

[34] V. V. Vyazovskiy, U. Olcese, E. C. Hanlon, Y. Nir, C. Cirelli, and G. Tononi, "Local sleep in awake rats," Nature, vol. 472, no. 7344, pp. 443-447, 2011.

[35] B. A. Klein, A. Klein, M. K. Wray, U. G. Mueller, and T. D. Seeley, "Sleep deprivation impairs precision of waggle dance signaling in honey bees," Proceedings of the National Academy of Sciences of the United States of America, vol. 107, no. 52, pp. 22705-22709, 2010.

[36] M. B. Andersson, Sexual Selection, Princeton University Press, Princeton, NJ, USA, 1994.

[37] J. A. Lesku, N. C. Rattenborg, M. Valcu et al., "Adaptive sleep loss in polygynous pectoral sandpipers," Science, vol. 337, no. 6102, pp. 1654-1658, 2012.

[38] R. Shumaker, S. Wich, and L. Perkins, "Reproductive life history traits of female orangutans (Pongo spp.)," in Primate Reproductive Aging, S. Atsalis, Margulis S. W., and P. R. Hof, Eds., vol. 36 of Interdisciplinary Topics in Gerontology, pp. 147161, Karger, Basel, Switzerland, 2008.

[39] D. R. Samson, Orangutan (Pongo pygmaeus) sleep architecture: testing the cognitive function of sleep and sleeping platforms in the Hominidae [Ph.D. thesis], Indiana University, Bloomington, Ind, USA, 2013.

[40] D. R. Samson and R. S. Shumaker, "Documenting orang-utan sleep architecture: sleeping platform complexity increases sleep quality in captive Pongo," Behaviour, vol. 150, pp. 845-861, 2013.

[41] D. R. Samson and R. W. Shumaker, "Pre-sleep and sleeping platform construction behavior in captive orangutans (Pongo spp.)," In press.

[42] J. Altmann, "Observational study of behavior: sampling methods," Behaviour, vol. 49, no. 3-4, pp. 227-266, 1974.

[43] R. Delgado, "Signal content and function in the long calls of wild male orangutans (Pongo pygmaeus)," American Journal of Primatology, vol. 60, p. 53, 2003.

[44] M. A. Carskadon and W. C. Dement, "Normal human sleep: an overview," in Principles and Practice in Sleep Medicine, M. H. Kryger, T. Roth, and W. C. Dement, Eds., pp. 13-23, Elsevier Saunders, Philadelphia, Pa, USA, 4th edition, 2005.

[45] J. F. Healey, Statistics: A Tool for Social Research, Thomson Wadsworth, Belmont, Mass, USA, 8th edition, 2009.

[46] J. J. McKenna, "Sudden infant death syndrome in cross-cultural perspective: is infant-parent cosleeping protective?" Annual Review of Anthropology, vol. 25, no. 1, pp. 201-216, 1996.

[47] J. J. McKenna, "Culture, infant sleep, and SIDS: an experiment in evolutionary medicine," American Journal of Human Biology, vol. 9, pp. 146-146, 1997.

[48] J. J. McKenna, H. L. Ball, and L. T. Gettler, "Mother-infant cosleeping, breastfeeding and sudden infant death syndrome: what biological anthropology has discovered about normal infant sleep and pediatric sleep medicine," American Journal of Physical Anthropology, vol. 45, pp. 133-161, 2007.

[49] R. Reimão, J. C. Souza, C. E. V. Gaudioso et al., "Nocturnal sleep pattern in Native Brazilian Terena adults," Arquivos de NeuroPsiquiatria, vol. 58, no. 2, pp. 233-238, 2000.

[50] C. M. Worthman and M. K. Melby, Eds., Toward a Comparative Developmental Ecology of Human Sleep, Cambridge University Press, Cambridge, Mass, USA, 2002.

[51] C. M. Worthman, Ed., After Dark: The Evolutionary Ecology of Human Sleep, Oxford University Press, Oxford, UK, 2008.

[52] A. R. Ekirch, "Sleep we have lost: pre-industrial slumber in the British Isles," The American Historical Review, vol. 106, no. 2, pp. 343-386, 2001.

[53] K. Zamma, "What makes wild chimpanzees wake up at night?" Primates, vol. 55, no. 1, pp. 51-57, 2013.

[54] B. Fruth and G. Hohmann, "Nest building behavior in the great apes: the great leap forward?" in Great Ape Societies, L. F. Marchant and T. Nishida, Eds., pp. 225-240, Cambridge University Press, Cambridge, UK, 1996.

[55] F. Marlowe, The Hadza: Hunter-Gatherers of Tanzania, University of California Press, Berkeley, Calif, USA, 2010.

[56] K. Hill and A. M. Hurtado, Ache Life History: The Ecology and Demography of a Foraging People, Aldine de Gruyter, New York, NY, USA, 1996. 

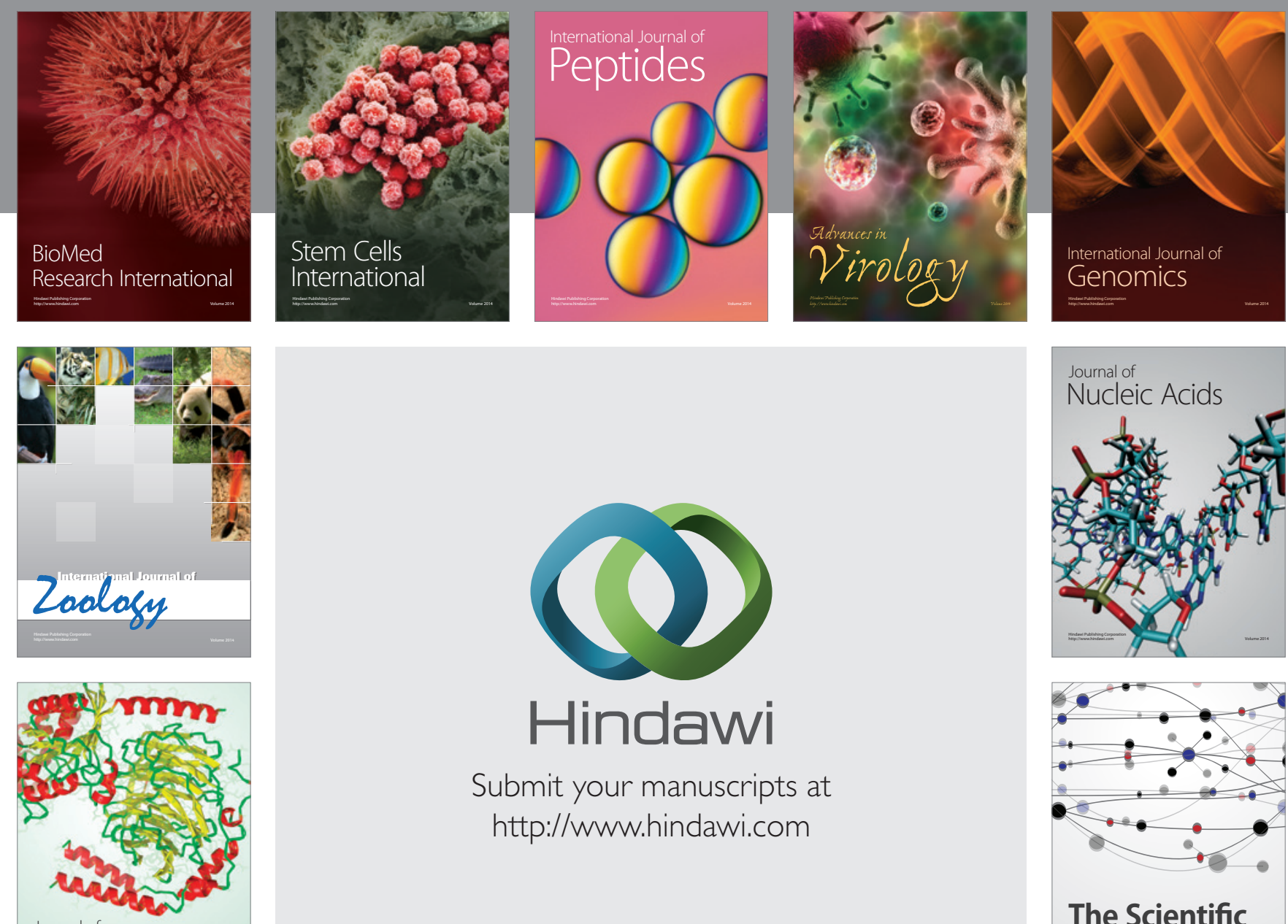

Submit your manuscripts at

http://www.hindawi.com

Journal of
Signal Transduction
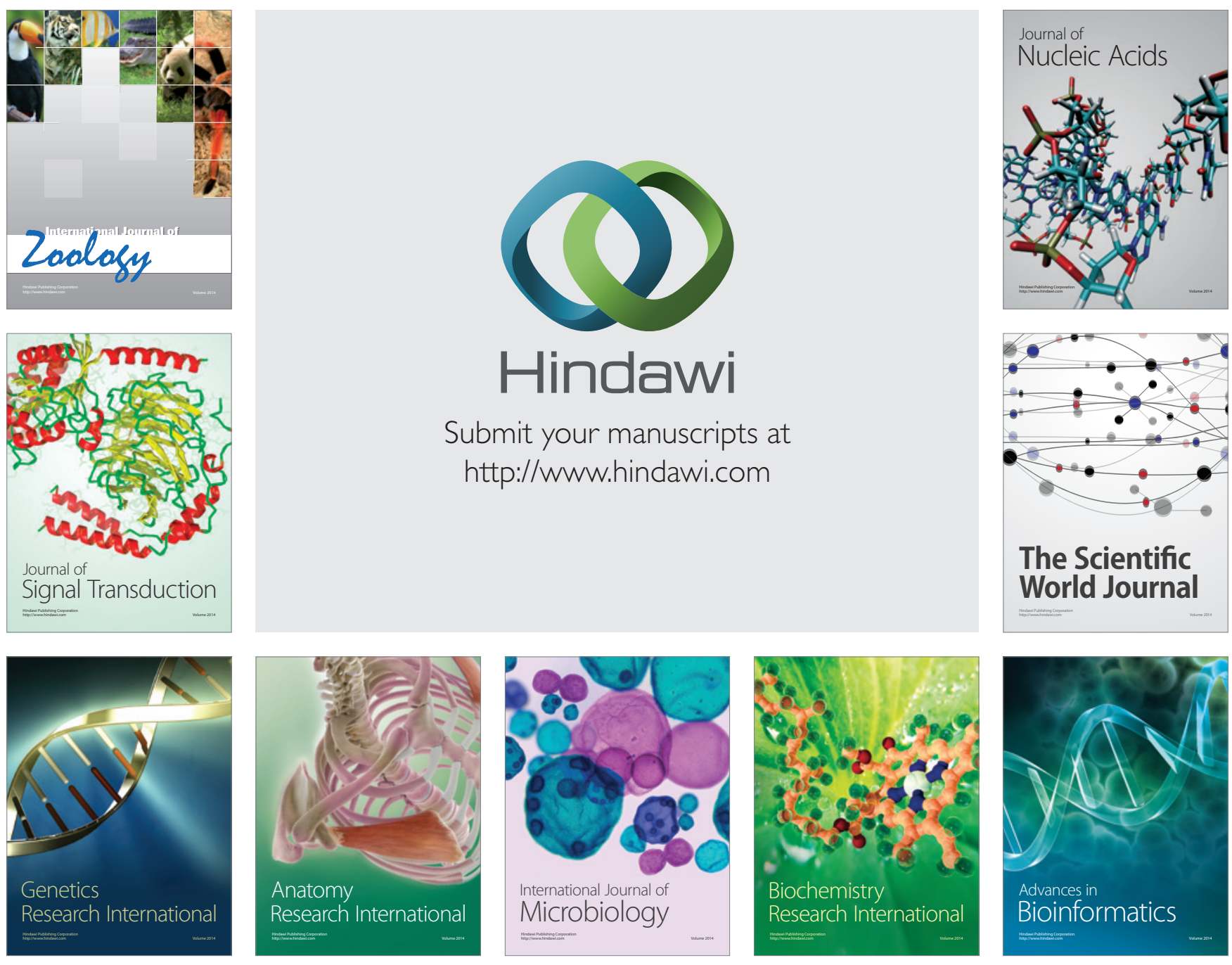

The Scientific World Journal
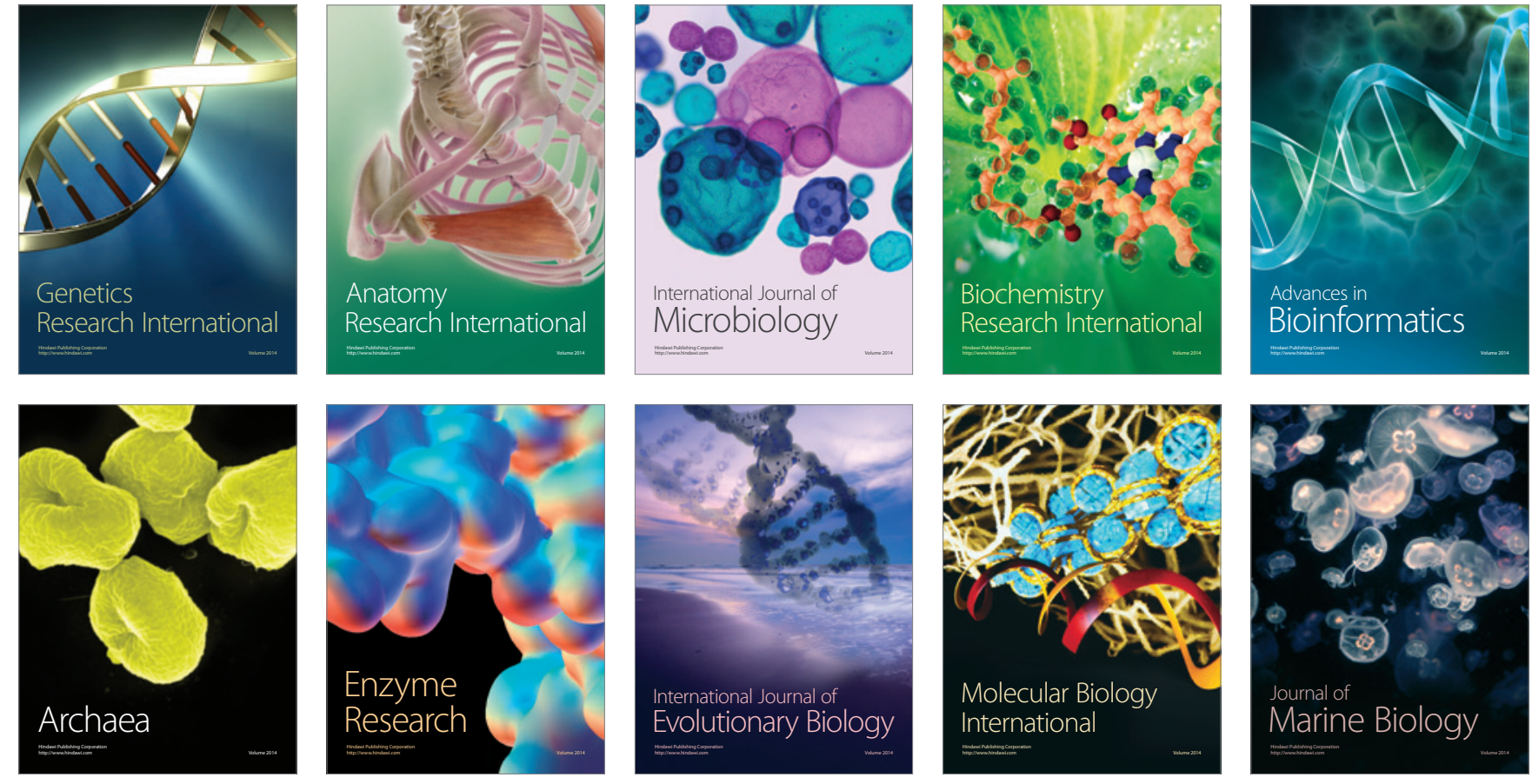